\title{
COLLECTIVE STATES FROM RANDOM INTERACTIONS
}

\author{
R. BIJKER ${ }^{1}$ and A. FRANK ${ }^{1,2}$ \\ ${ }^{1}$ ICN-UNAM, A.P. 70-543, 04510 México, D.F., México \\ 2 CCF-UNAM, A.P. 139-B, Cuernavaca, Morelos, México
}

\begin{abstract}
The anharmonic vibrator and rotor regions in nuclei are investigated in the framework of the interacting boson model using an ensemble of random one- and twobody interactions. Despite the randomness of the interactions (in sign and size) we find a predominance of $L^{P}=0^{+}$ground states and strong evidence for the occurrence of both vibrational and rotational band structure.
\end{abstract}

\section{Introduction}

In empirical studies of medium and heavy even-even nuclei very regular features have been observed, such as the tripartite classification of nuclear structure into seniority, anharmonic vibrator and rotor regions 1 . In each of these three regimes, the energy systematics is extremely robust, and the transitions between different regions occur very rapidly, typically with the addition or removal of only one or two pairs of nucleons. These robust features suggest that there exists an underlying simplicity of low-energy nuclear structure never before appreciated. In order to address this point we present the results of a study of the systematics of collective levels in the framework of the interacting boson model (IBM) with random interactionse.

\section{Randomly interacting bosons}

In the IBM, collective excitations in nuclei are described in terms of a system of $N$ interacting monopole and quadrupole bosons 3 . We consider the most general one- and two-body Hamiltonian $H=H_{1} / N+H_{2} / N(N-1)$. The two one-body and seven two-body matrix elements of the IBM are chosen independently from a Gaussian distribution of random numbers with zero mean and variance $v^{2}$, such that the ensemble is invariant under orthogonal basis transformations. In order to remove the $N$ dependence of the many-body matrix elements, we have scaled $H_{1}$ by $N$ and $H_{2}$ by $N(N-1)$. In all calculations we take $N=16$ bosons and 1000 runs. For each set of randomly generated one- and two-body matrix elements we calculate the entire energy spectrum and the $B(E 2)$ values between the yrast states.

Just as for the nuclear shell model with random interactions 咽, we find a predominance of $L^{P}=0^{+}$ground states. In $63.4 \%$ of the runs the ground 
Table 1: Energies and $B(E 2)$ values in the dynamical symmetry limits of the IBM 3 . In the $U(5)$ and $S O(6)$ limits we show the result for the leading order contribution to the rotational spectra.

\begin{tabular}{|ccc|}
\hline & $R=\frac{E\left(4^{+}\right)-E\left(0^{+}\right)}{E\left(2^{+}\right)-E\left(0^{+}\right)}$ & $\frac{B\left(E 2 ; 4^{+} \rightarrow 2^{+}\right)}{B\left(E 2 ; 2^{+} \rightarrow 0^{+}\right)}$ \\
\hline & & $\frac{2(N-1)}{N}$ \\
$S(5)$ & 2 & $\frac{10(N-1)(N+5)}{7 N(N+4)}$ \\
$S U(6)$ & $\frac{5}{2}$ & $\frac{10(N-1)(2 N+5)}{7 N(2 N+3)}$ \\
\hline
\end{tabular}

state has $L^{P}=0^{+}$, followed by the state with the maximum value of the angular momentum $L^{P}=32^{+}$with $16.7 \%$ and $L^{P}=2^{+}$with $13.8 \%$.

For the cases with a $0^{+}$ground state we calculate the probability distribution $P(R)$ of the energy ratio of the yrast states $R=\left[E\left(4^{+}\right)-E\left(0^{+}\right)\right] /\left[E\left(2^{+}\right)-\right.$ $\left.E\left(0^{+}\right)\right]$. This energy ratio has characteristic values of $R \approx 1,2$ and $10 / 3$ for the seniority, vibrational and rotational regions, respectively. Fig. 11 shows a remarkable result: $P(R)$ has two very pronounced peaks, one at $R \sim 1.95$ and a narrower one at $R \sim 3.35$ 2 . These values correspond almost exactly to the harmonic vibrator and rotor values of 2 and 10/3 (see Table 1).

Energies by themselves are not sufficient to decide whether or not there exists a collective structure. Levels belonging to a collective band are connected by strong electromagnetic transitions. In Table 1 we show the energy ratio $R$ and the ratio of $B(E 2)$ values of the $4^{+} \rightarrow 2^{+}$and $2^{+} \rightarrow 0^{+}$transitions for the three symmetry limits of the IBMB. In the large $N$ limit, the ratio of $B(E 2)$ values is 2 for the harmonic vibrator and $10 / 7$ both for the $\gamma$-unstable rotor and the axially symmetric rotor. In Fig. 2 we show a correlation plot between the ratio of $B(E 2)$ values and the energy ratio $R$. There is a strong correlation between the first peak in the energy ratio and the vibrator value for the ratio of $B(E 2)$ values (the concentration of points in this region corresponds to about $50 \%$ of all cases), and for the second peak and the rotor value (about $25 \%$ of all cases) 1 .

A subsequent study 6 of the dependence of these collective features on the number of bosons $N$ and the rank $k$ of the interactions has shown that the vibrational and rotational band structures appear gradually as a function of $N / k$. For $N \sim k$ there is little or no evidence for such bands. For larger values 


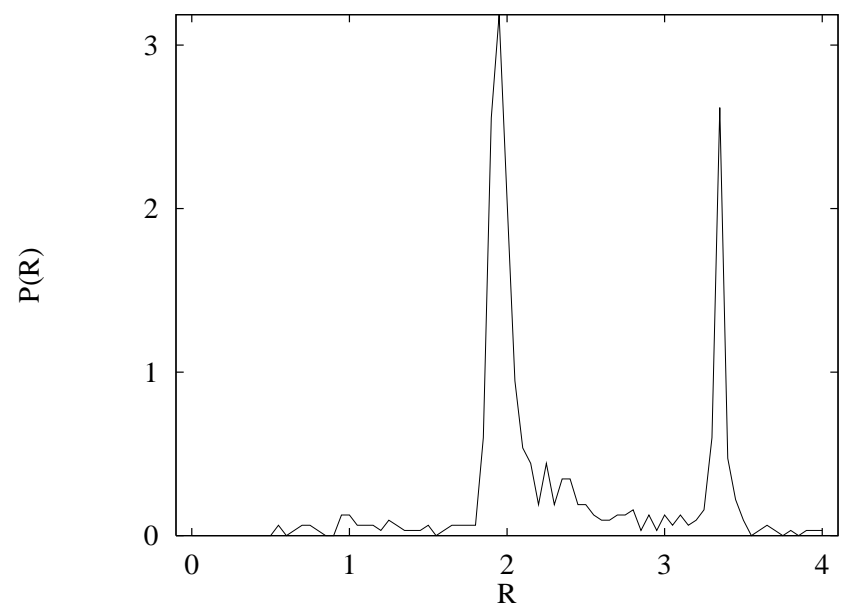

Figure 1: Probability distribution $P(R)$ of the energy ratio $R=\left[E\left(4^{+}\right)-E\left(0^{+}\right)\right] /\left[E\left(2^{+}\right)-\right.$ $\left.E\left(0^{+}\right)\right]$with $\int P(R) d R=1$ in the IBM with random one- and two-body interactions. The number of bosons is $N=16$.

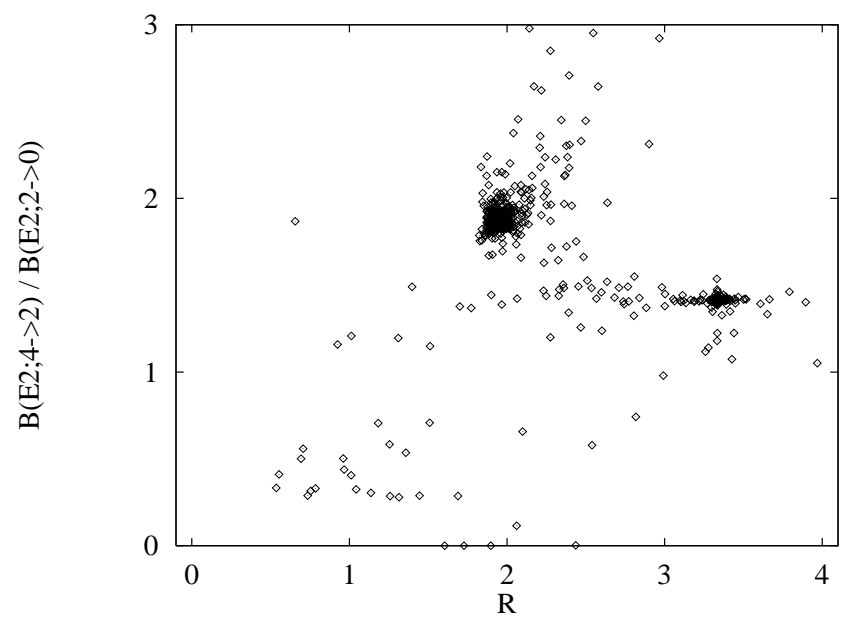

Figure 2: Correlation between ratios of $B(E 2)$ values and energies in the IBM with random one- and two-body interactions. 
of $N$ we first see the development of vibrational structure, followed later by the appearance of rotational bands. If $N$ increases further these band structures become more and more pronounced.

\section{Conclusions}

A study of the IBM with random ensembles of one- and two-body Hamiltonians has shown that despite the randomness of the interactions the ground state has $L^{P}=0^{+}$in $63.4 \%$ of the cases. For this subset, the analysis of energies and quadrupole transitions shows strong evidence for the occurrence of both vibrational and rotational band structure. The inclusion of random three-body interactions does not significantly change the basic properties.

These regular features arise from a much wider class of Hamiltonians than are usually considered to be 'realistic', and represent general and robust properties of the many-body dynamics, which enters via the reduction formulas for the $N$-body matrix elements of $k$-body interactions. Since the structure of the model space is completely determined by the corresponding degrees of freedom, these results emphasize the importance of the selection of the relevant degrees of freedom.

A similar situation has been observed in the context of the nuclear shell model with respect to the pairing properties $\mathrm{U}$.

\section{Acknowledgements}

This work was supported in part by DGAPA-UNAM under project IN101997, and by CONACyT under projects 32416-E and 32397-E.

\section{References}

1. N.V. Zamfir, R.F. Casten and D.S. Brenner, Phys. Rev. Lett. 72, 3480 (1994).

2. R. Bijker and A. Frank, Phys. Rev. Lett. 84, 420 (2000).

3. F. Iachello and A. Arima, The interacting boson model (Cambridge University Press, 1987).

4. C.W. Johnson, G.F. Bertsch and D.J. Dean, Phys. Rev. Lett. 80, 2749 (1998).

5. R. Bijker, A. Frank and S. Pittel, Phys. Rev. C 60, 021302 (1999).

6. R. Bijker and A. Frank, Phys. Rev. C, in press. Preprint nuclth/0004002.

7. C.W. Johnson, G.F. Bertsch, D.J. Dean and I. Talmi, Phys. Rev. C 61, 014311 (2000). 